\title{
Cosmology and thermodynamics of FLRW universe with bulk viscous stiff fluid
}

\author{
Titus K. Mathew ${ }^{\mathrm{a}}$, M. B. Aswathy, M. Manoj \\ Department of Physics, Cochin University of Science and Technology, Cochin, India
}

Received: 10 June 2014 / Accepted: 20 November 2014 / Published online: 9 December 2014

(c) The Author(s) 2014. This article is published with open access at Springerlink.com

\begin{abstract}
We consider a cosmological model dominated by a stiff fluid with a constant bulk viscosity. We classify all the possible cases of the universe predicted by the model and analyze the scale factor and the density as well as the curvature scalar. We find that when the dimensionless constant bulk viscous parameter is in the range $0<\bar{\zeta}<6$ the model begins with a big bang and makes a transition from the decelerating expansion epoch to an accelerating epoch and then tends to the de Sitter phase as $t \rightarrow \infty$. The transition into the accelerating epoch would be in the recent past when $4<\bar{\zeta}<6$. For $\bar{\zeta}>6$ the model does not have a big bang and shows an increase in the fluid density and scalar curvature as the universe expands which eventually saturates as the scale factor $a \rightarrow \infty$ in the future. We have analyzed the model with statefinder diagnostics and find that the model is different from the $\Lambda \mathrm{CDM}$ model but approaches the $\Lambda \mathrm{CDM}$ point as $a \rightarrow \infty$. We have also analyzed the status of the generalized second law of thermodynamics with an apparent horizon as the boundary of the universe and found that the law is generally satisfied when $0 \leq \bar{\zeta}<6$, and for $\bar{\zeta}>6$ the law is satisfied when the scale factor is larger than a minimum value.
\end{abstract}

\section{Introduction}

Recent studies on the current acceleration of the universe using type Ia supernovae data [1-3] have shown that about $72 \%$ of the energy density of the universe is in the form of an exotic component, capable of producing a negative pressure, called dark energy. Cosmological data from a wide range of other sources- the cosmic microwave background radiation [4,5], baryon acoustic oscillations [6], cluster gas fractions [7], and gamma ray bursts [8,9] have all confirmed this conclusion. Concerning the remaining part of the energy density, it was concluded even before the discovery of dark energy

\footnotetext{
a e-mail: titus@cusat.ac.in
}

that $23 \%$ of it consists of weakly interacting matter called dark matter. The evidence for this comes from a variety of observational tests including weak [10] and strong [11] lensing, large scale structure [12], as well as from supernovae and cosmic microwave background [13-15] studies. In spite of the fact that all these observational data establish the existence of the components like dark matter and dark energy, the existence of other exotic fluid components has not been ruled out. For example several models predict the existence of an exotic component called dark radiation in the universe [16]. Another exotic fluid which is predicted by several models is a stiff fluid, a fluid with an equation of state $p_{\mathrm{s}}=\rho_{\mathrm{s}}$, where $p_{\mathrm{S}}$ and $\rho_{\mathrm{S}}$ are the normal pressure and density of the stiff fluid, respectively. The equation of state parameter of this fluid assumes the largest value (equal to 1) consistent with causality because the speed of sound in this fluid is equal to the speed of light.

The model with a stiff fluid was first studied by Zeldovich [17]. In recent years a large number of models have been proposed for studying various cosmological properties of stiff fluids. In certain models with self-interacting dark matter components, the self-interaction between the dark matter particles is characterized by the exchange of vector mesons via minimal coupling. In such models the self-interaction energy is shown to behave like a stiff fluid [18]. A stiff fluid has also been considered in certain cosmological models based on Horava-Lifshitz gravity. In Horava-Lifshitz gravity theories a "detailed balancing" condition was imposed as a convenient simplification and the usefulness of this detailed balancing condition was discussed in Refs. [19-21]. The stiff fluid appears in such models when this detailed balancing condition is relaxed [22-25]. Cosmological models with a stiff fluid, based on Horava-Lifshitz gravity, have been studied in Refs. [26,27]. The existence of a stiff fluid have also been found as exact non-singular solutions in certain inhomogeneous cosmological models [28-31]. The relevance of the stiff fluid equation of state to the matter content of the 
universe in the early stage of the universe was investigated in Ref. [32]. In Ref. [33], the authors deal with some symmetry related properties of a bulk viscous stiff fluid where they argue that global conformal symmetry is incompatible with a non-vanishing entropy production, but conformal symmetries related to a specific internal interaction that macroscopically corresponds to negative pressure may be compatible with the production of entropy. In such cases one can separate the total pressure into a normal pressure and an effective negative pressure which satisfies the equation of state of the stiff fluid.

The decrease in the density of stiff fluid in the universe is found to be faster than that of radiation and matter; hence its effect on the expansion would be larger in the initial stage of the universe. Primordial nucleosynthesis is an event that took place in the early phase of the universe and a limit on the density of the stiff fluid can be obtained from big bang nucleosynthesis constraints as in Ref. [34].

In a homogeneous and isotropic universe, bulk viscosity is the unique viscous effect capable of modifying the background dynamics. From a theoretical point of view, bulk viscosity arises in a system due to its deviations from local thermodynamic equilibrium [35]. In cosmology, bulk viscosity arises as an effective pressure, restoring the system to its thermal equilibrium, which was broken when universe expanded too fast so that the system did not get enough time to restore the local thermal equilibrium [36-39]. Several years ago, before the discovery of the present acceleration of the universe, it has been proposed, in the context of the inflationary scenario of the early universe that a bulk viscous fluid can produce acceleration in the expansion of the universe [4046]. Recently investigations were made of the possibility of bulk viscous matter causing the recent acceleration of the universe [47-50].

In the present work we study a stiff fluid dominated cosmological model with bulk viscosity. We consider a universe with effectively one component, the stiff fluid. Such a one component description may not comply with the most general realistic situation. However, this will help us in understanding the essential features of a universe which is dominated by a bulk viscous stiff fluid. We assume a stiff fluid with the equation of state $p_{\mathrm{s}}=\rho_{\mathrm{s}}$ and the bulk viscosity characterized by a constant viscosity coefficient which is the simplest parametrization for the bulk viscosity. We derive the Hubble parameter, density, equation of state, and deceleration parameter, and we analyze their behavior with regard to further possibilities including the recent acceleration of the universe. The paper is organized as follows. In Sect. 2 we give the basic equations of the FLRW (Friedmann-LemaitreRobertson-Walker) universe and derive the general equation for the Hubble parameter in a bulk viscous stiff fluid dominated universe. We classify the different cases depending on the value of the bulk viscous parameter and analyze the evolu- tion of various cosmological parameters. Section 3 contains the statefinder diagnosis of the model. In Sect. 4 we present the status of the generalized second law of thermodynamics. This is followed by the presentation of our conclusions in Sect. 5 .

\section{Stiff fluid with bulk viscosity}

Stiff fluid cosmological models have generated interest because in these fluids the speed of light is equal to the speed of sound and the governing equations have the same characteristics as that of the gravitational field [51]. The equation of state of the stiff fluid is given as [17]

$p_{\mathrm{s}}=\rho_{\mathrm{s}}$.

This equation of state corresponds to a special case of the models investigated by Masso and others [52].

In cosmological models the effect of bulk viscosity can be shown to be an added correction to the net pressure $p_{\mathrm{s}}^{\prime}$ as

$p_{\mathrm{s}}^{\prime}=p_{\mathrm{s}}-3 \zeta H$,

where $\zeta$ is the constant coefficient of viscosity and $H$ is the Hubble parameter. The form of the above equation was originally proposed by Eckart [53] in the context of relativistic dissipative processes occurring in thermodynamic systems which have gone out of local thermal equilibrium. Later Landau and Lifshitz [54] proposed an equivalent formulation. However, Eckart's theory has got the shortcoming that it describes all the equilibria as unstable [55] and signals can propagate through the fluid with superluminal velocities [56]. Later Israel and Stewart $[57,58]$ proposed a more general theory which avoids these problems and from which Eckart theory appears as the first order limit. However, because of the simple form of Eckart theory, it has been used by several authors to characterize the bulk viscous fluid. For example the Eckart approach has been used in models explaining the recent acceleration of the universe with a bulk viscous fluid [59-63]. Moreover, Hiscock et al. [64] have shown that Eckart theory is to be favored over the Israel-Stewart model in explaining the inflationary acceleration of FLRW universe with bulk viscous fluid. These motivate the use of Eckart's results, especially when one tries to look at the phenomenon of the recent acceleration of the universe. At this point one may also take note of the more general formulation than the Israel-Stewart by Pavon et al. dealing with the thermodynamic equilibrium as in Ref. [65]

We consider the flat FLRW universe favored by the recent WMAP observation [66] with the scale factor

$$
\mathrm{d} s^{2}=-\mathrm{d} t^{2}+a^{2}(t)\left(\mathrm{d} r^{2}+r^{2} \mathrm{~d} \theta^{2}+r^{2} \sin \theta \mathrm{d} \phi^{2}\right),
$$


where $a(t)$ is the scale factor, $t$ is the cosmic time, and $(r, \theta, \phi)$ are the comoving coordinates. The corresponding dynamical equations are

$H^{2}=\frac{\rho}{3}$

$2 \frac{\ddot{a}}{a}+\left(\frac{\dot{a}}{a}\right)^{2}=p^{\prime}$

and the conservation equation is

$\dot{\rho}+3 H\left(\rho+p^{\prime}\right)=0$,

where we have adopted the standard units convention, $8 \pi G=1$; an over-dot represents a derivative with respect to cosmic time. From the dynamical equations (4) and (5), we can formulate a first order differential equation for the Hubble parameter by using Eqs. (1), (2), and (6) as

$\dot{H}=\frac{3 H}{2}(\zeta-2 H)$

The above equation can be expressed in terms of the variable $x=\log a$, suitably integrated and the final result can be written in terms of the scale factor as

$H=\frac{H_{0}}{6}\left[\bar{\zeta}+(6-\bar{\zeta}) a^{-3}\right]$,

where $\bar{\zeta}=3 \zeta / H_{0}$ is the dimensionless bulk viscous coefficient, $H_{0}$ is the present value of the Hubble parameter, and we have made the assumption that the present value of the density parameter of the stiff fluid $\Omega_{s 0}=1$ for a stiff fluid dominated universe.

\subsection{Classification and evolution of the bulk viscous stiff fluid dominated model}

The equation for the Hubble parameter shows that for different values of the viscosity coefficient $\bar{\zeta}$ we get different models. In this section we classify different models of the universe which arise due to the different values of the dimensionless viscosity coefficient. We analyze the behavior of the scale factor, the density, and other parameters in these different cases.

\subsubsection{Case $1: \bar{\zeta}=0$}

This corresponds to the universe dominated by a stiff fluid without bulk viscosity. From Eq. (8) the Hubble parameter becomes $H=H_{0} a^{-3}$. From the dynamical equation (4) the corresponding density of the stiff fluid follows the relation

$\rho_{\mathrm{s}} \propto a^{-6}$.

This shows that the density of the non-viscous stiff fluid decays more rapidly than the non-relativistic matter or radiation in a FLRW universe, which implies that the effect of the stiff fluid on the expansion of the universe would be larger at early times. So the limit on the density of the stiff fluid can be obtained by considering its effect on the big bang nucleosynthesis. Dutta et al. [34] made an investigation in this regard and found that the change in the primordial abundance of helium- 4 is proportional to the ratio $\rho_{\mathrm{S}} / \rho_{\mathrm{R}}$, where $\rho_{\mathrm{R}}$ is the radiation density. Consequently they found a limit on the non-viscous stiff fluid density as $\rho_{\mathrm{S}} / \rho_{\mathrm{R}}<30$ when the temperature of the universe was around $10 \mathrm{MeV}$.

The evolution of the scale factor can be obtained by integrating the Hubble parameter as

$a(t)=\left(3 H_{0}\left(t-t_{0}\right)+1\right)^{1 / 3}$.

The second order derivative of the scale factor with time is

$\frac{\mathrm{d}^{2} a}{\mathrm{~d} t^{2}}=-\frac{2 H_{0}^{2}}{\left(3 H_{o}\left(t-t_{0}\right)+1\right)^{5 / 3}}$.

This shows that the universe will undergo an eternal deceleration in this case.

The behavior of the density following from Eq. (9) reveals that as the scale factor $a(t) \rightarrow 0$, the density $\rho_{\mathrm{s}} \rightarrow \infty$. This implies the existence of a singularity at the beginning of the universe. This fact can be further verified by calculating the curvature scalar for the flat FLRW universe using the equation [67]

$R=\left(\frac{\ddot{a}}{a}+H^{2}\right)$.

Using the equation for the Hubble parameter and its time derivative it can easily be shown that $R \sim H^{2}$, which according to the equation $H=H_{0} a^{-3}$ implies that the curvature scalar $R \rightarrow \infty$ as $a \rightarrow 0$ at the origin, confirming the presence of the initial singularity. So it can be concluded that in this case the universe had a big bang. The time elapsed since the big bang, $t_{\mathrm{B}}$, is found to be

$t_{\mathrm{B}}=t_{0}-\frac{1}{3 H_{0}}$.

Also it is evident from the behavior of the density that, as $a(t) \rightarrow \infty$ the density $\rho_{\mathrm{s}} \rightarrow 0$. In this respect apart from the difference in the dependence on the scale factor, the general behavior of the non-viscous stiff fluid is the same as that of non-relativistic matter or relativistic radiation.

\subsubsection{Case 2: $0<\bar{\zeta}<6$}

The Hubble parameter is given by Eq. (8). Following the dynamical equations the density of the bulk viscous stiff fluid in this case is given by

$\rho_{\mathrm{s}}=3\left(\frac{H_{0}}{6}\left(\bar{\zeta}+(6-\bar{\zeta}) a^{-3}\right)\right)^{2}$.

The evolution of the density with scale factor is given in Fig. 1. This equation shows that as the scale factor $a(t) \rightarrow 0$ 


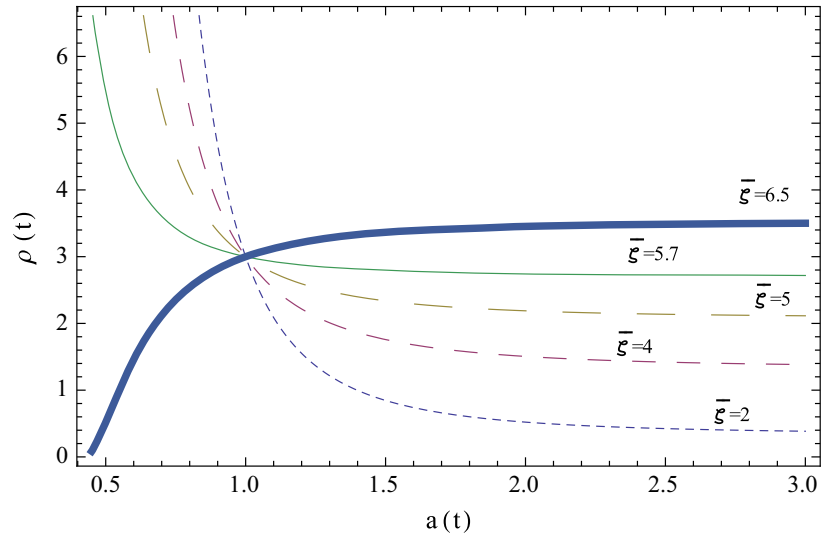

Fig. 1 Evolution of the density $\rho_{\mathrm{s}}$ with scale factor $a(t)$

the density $\rho_{\mathrm{s}} \rightarrow \infty$, indicating that there is a singularity at the origin. The presence of the singularity is further confirmed by calculating the curvature scale using Eq. (12) and it is

$R=\frac{3 H \bar{\zeta}}{2}-H^{2}$,

which shows that $R \rightarrow \infty$ as $a(t) \rightarrow 0$, confirming the presence of the initial singularity. So the model of the universe in this case does have a big bang.

For finding the scale factor, Eq. (8) can be put in the form

$\frac{\mathrm{d} a^{3}}{\mathrm{~d} t}-\frac{H_{0} \bar{\zeta}}{2} a^{3}=\frac{H_{0}(6-\bar{\zeta})}{2}$,

which can be integrated to obtain the scale factor as

$a(t)=\left(\frac{\bar{\zeta}-6+6 \exp \left(\bar{\zeta} H_{o}\left[t-t_{0}\right] / 2\right)}{\bar{\zeta}}\right)^{1 / 3}$.

This equation for the scale factor reveals that the time elapsed since the big bang is

$t_{\mathrm{B}}=t_{0}+\frac{2}{H_{0} \bar{\zeta}} \ln \left(\frac{6-\bar{\zeta}}{6}\right)$,

and hence the age of the universe since the big bang is

$t_{0}-t_{\mathrm{B}}=-\frac{2}{H_{0} \bar{\zeta}} \ln \left(\frac{6-\bar{\zeta}}{6}\right)$.

Taking $H_{0}=100 h \mathrm{~km} / \mathrm{s} / \mathrm{Mpc}$, with $h=0.74$, the age of the universe evaluated as per the above equation is around $13.8 \mathrm{Gyr}$ for $\bar{\eta}=5.7$, a value which is very close to that predicted by the CMB anisotropy data [69].

A plot of the evolution of the scale factor is given in Fig. 2. The scale factor Eq. (17) shows that as $t \rightarrow \infty$ the scale factor approaches a form like that of the de Sitter universe,

$a(t) \rightarrow \exp \left(\bar{\zeta} H_{0}\left[t-t_{0}\right] / 2\right)$.

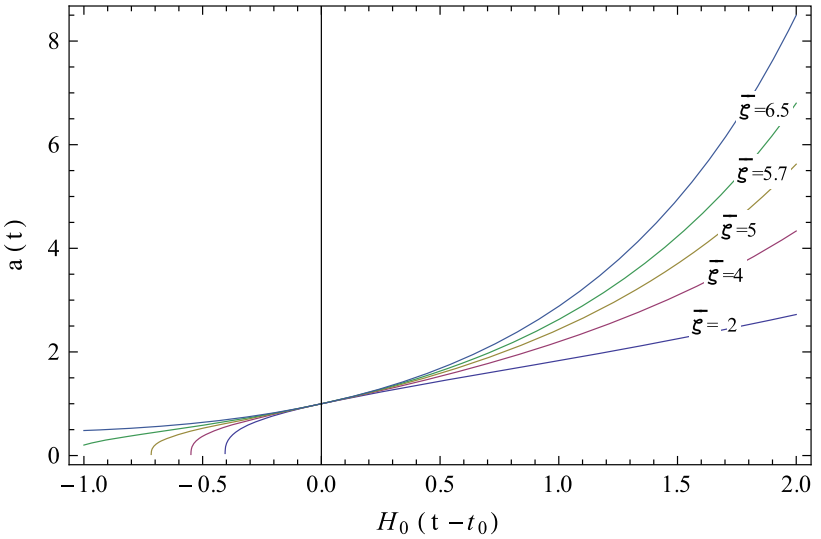

Fig. 2 Evolution of the scale factor $a(t)$ with $H_{0}\left(t-t_{0}\right)$ for different values of dimensionless bulk viscous parameter $\bar{\zeta}$

In the early stages of the evolution of the universe, when $\bar{\zeta} H_{0}\left[t-t_{0}\right] / 2<1$, the scale factor can be approximated as

$a(t) \sim\left(1+3 H_{0}\left[t-t_{0}\right]\right)^{1 / 3}$.

These equations of the scale factor at the respective limits show that the universe had an earlier deceleration phase followed by an acceleration phase in the later stage of the evolution. This means that the bulk viscous stiff fluid behaves almost like cold dark matter in the past and behaves like dark energy in the later phase of the accelerating universe, indicating that this model can naturally unify the dark matter and dark energy at least at the background level. The value of the scale factor or the red shift at which the transition from the decelerated to the accelerated expansion occurs depends on the viscosity coefficient $\bar{\zeta}$ as shown below. From the Hubble parameter we can calculate the derivative of $\dot{a}$ with respect $a(t)$ as

$\frac{\mathrm{d} \dot{a}}{\mathrm{~d} a}=\bar{\zeta}-2(6-\bar{\zeta}) a^{-3}$.

Equating this to zero, we obtain the transition scale factor as [49]

$a_{\mathrm{T}}=\left(\frac{2(6-\bar{\zeta})}{\bar{\zeta}}\right)^{1 / 3}$

and the corresponding transition redshift is

$z_{\mathrm{T}}=\left(\frac{\bar{\zeta}}{2(6-\bar{\zeta})}\right)^{1 / 3}-1$

From Eqs. (23) and (24) it is clear that for $\bar{\zeta}=4$ the transition from the decelerated phase to the accelerated phase occurs at $z_{\mathrm{T}}=0, a_{\mathrm{T}}=1$, which corresponds to the present stage of the universe. In the range $0<\bar{\zeta}<4$ the transition between the decelerated and the accelerated phase takes place in the future corresponds to $z_{\mathrm{T}}<0, a_{\mathrm{T}}>1$. The transition takes place in the past of the universe $\left(z_{\mathrm{T}}>0, a_{\mathrm{T}}<1\right)$ when $4<\bar{\zeta}<6$. When $\bar{\zeta}=0$ the value of $z_{\mathrm{T}}$ becomes -1 


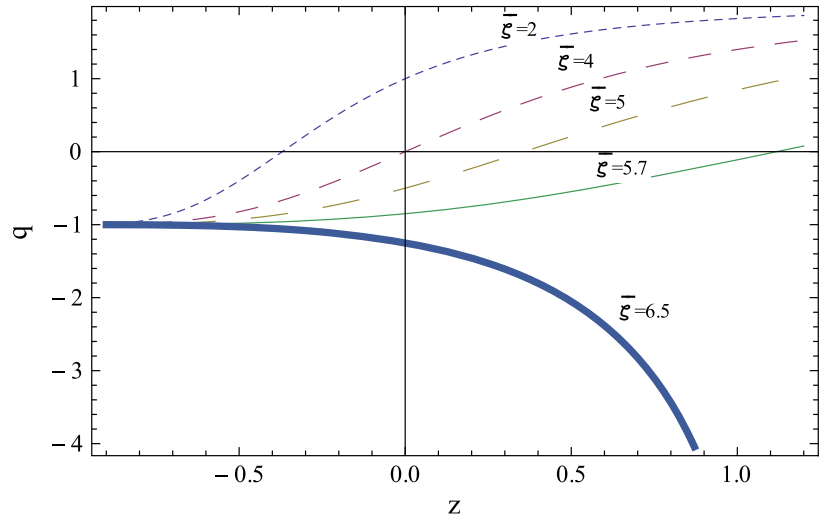

Fig. 3 Evolution of the deceleration parameter $q$ with redshift $z$

and the value of the scale factor $a_{\mathrm{T}}$ becomes infinity in the future, this implies that there is no transition to accelerated expansion within a finite time, and the universe is always decelerating. For $\bar{\zeta}=6$ the transition takes place at a time corresponding to $a_{\mathrm{T}} \rightarrow 0$, closer to the big bang.

As a further clarification of the conclusions in the above paragraph we evaluate the deceleration parameter and the equation of state parameter of the bulk viscous stiff fluid in this case. A positive value of the deceleration parameter characterizes a decelerating universe, while a negative value characterizes an accelerating universe. The deceleration parameter $q$ can be evaluated using the equation

$q=-1-\frac{\dot{H}}{H^{2}}$.

Using the Hubble parameter from Eq. (8), the deceleration parameter in terms of the redshift $z$ is

$q=-1-\frac{3(\bar{\zeta}-6)(1+z)^{3}}{\bar{\zeta}+(6-\bar{\zeta})(1+z)^{3}}$

where we took $a=(1+z)^{-1}$. The evolution of the deceleration parameter is shown in Fig. 3. It is clear from the figure that the deceleration parameter $q \rightarrow-1$ in the far future of the evolution of the universe as $z \rightarrow-1$ for any positive value of the dimensionless bulk viscous parameter $\bar{\zeta}$.

The transition redshift $z_{\mathrm{T}}$ can be obtained by equating $q$ to zero, and it leads to Eq. (24). For $\bar{\zeta}=0$ the deceleration parameter will be 2 , corresponding to a universe dominated by a non-viscous stiff fluid. For $\bar{\zeta}=6$ the parameter $q=-1$ corresponds to the de Sitter phase. So for $0<\bar{\zeta}<6$ the deceleration parameter is always decreasing from $q(a=0)=2$ to $q(a=\infty)=-1$, with a transition from positive to negative values corresponding to the transition from deceleration to acceleration in the expansion of the universe. The deceleration parameter today, i.e. for $z=0$, is found to be

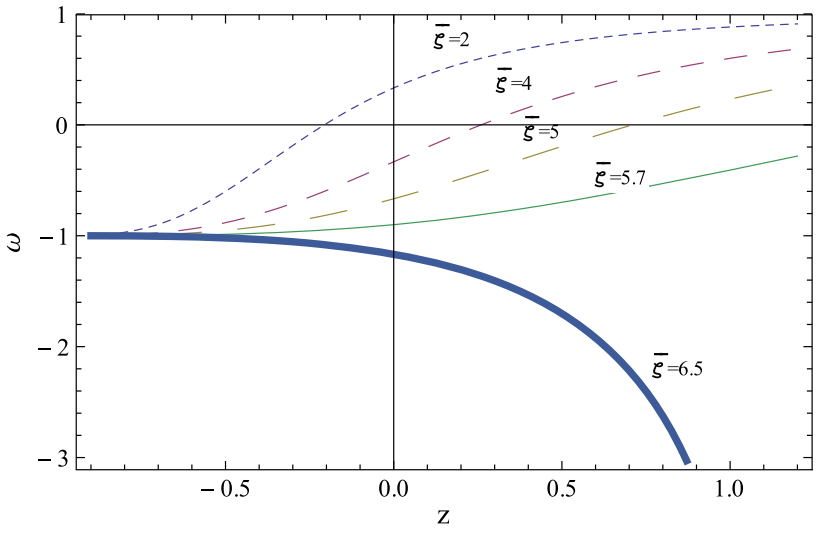

Fig. 4 Evolution of the equation of state parameter $\omega$ with redshift $z$

$q(a=1)=2-\frac{\bar{\zeta}}{2}$.

This agrees with our earlier results in Eqs. (23) and (24) that for $\bar{\zeta}=4$ the universe would enter the accelerating phase from the decelerated expansion at the present time. For $\bar{\zeta}<4$, corresponding to $q>0$, we have a decelerating universe today and for $\bar{\zeta}>4$ corresponding to $q<0$, we have an accelerating universe today. From the current observational results $[66,69]$, the present value of the deceleration parameter is around $-0.64 \pm 0.03$, from which the bulk viscous coefficient is seen to be $\bar{\zeta}>4$ for a universe dominated by a bulk viscous stiff fluid. This analysis shows that if universe is dominated by a bulk viscous stiff fluid, it can take the role of the conventional dark energy, causing the recent acceleration of the universe for a bulk viscous coefficient in the range $4<\bar{\zeta}<6$.

The evolution of the equation of state $\omega_{\mathrm{S}}$ of the stiff fluid with bulk viscosity can be studied by calculating it using the relation [70]

$\omega_{\mathrm{s}}=-1-\frac{1}{3} \frac{\mathrm{d} \ln h^{2}}{\mathrm{~d} x}$,

where $h=H / H_{0}$, the weighted Hubble parameter. Evaluating $\omega_{\mathrm{S}}$ in terms of the redshift $z$ gives

$\omega_{\mathrm{s}}=-1-\left(\frac{2(\bar{\zeta}-6)(1+z)^{3}}{\bar{\zeta}+(6-\bar{\zeta})(1+z)^{3}}\right)$.

The evolution of the equation of state is as shown in Fig. 4.

As $z \rightarrow-1,(a \rightarrow \infty)$ the equation of state parameter $\omega_{\mathrm{s}} \rightarrow-1$ in the future, which corresponds to the de Sitter universe, irrespective of the value of the viscosity coefficient. For $\bar{\zeta}=0$ the equation of state parameter becomes $\omega_{\mathrm{s}}=1$, implying the equation of state for the non-viscous stiff fluid, $p_{\mathrm{s}}=\rho_{\mathrm{s}}$. For $\bar{\zeta}=6$ the $\omega_{\mathrm{s}}$ becomes equal to -1 . In the range $0<\bar{\zeta}<6$ the equation of state varies from +1 to -1 , making possible a transition from positive to negative values. Even though a negative value of $\omega_{\mathrm{s}}$ leads to a negative 
pressure, for the universe to be in the accelerating phase we must have $\omega_{\mathrm{s}}<-1 / 3$.

The present value of $\omega_{\mathrm{s}}$ is found to be

$\omega_{\mathrm{S}}(a=1)=1-\frac{\bar{\zeta}}{3}$.

This equation reveals that $\omega_{\mathrm{s}}$ makes a transition from positive values to negative values at the present time if $\bar{\zeta}=3$. While considering the evolution of the $q(a=1)$ parameter, we have shown that $q$ makes a transition to negative values, giving a universe with an accelerated expansion for $\bar{\zeta}=4$. The negativity of the parameter $q$ implies that the universe is accelerating and at the same time the equation of state parameter must be less than $-1 / 3$ for the universe to be accelerated [68]. From Eq. (30) it is clear that $\omega_{\mathrm{s}}(a=1)<-1 / 3$ only for $\bar{\zeta} \geq 4$. The current observational value of the equation of state parameter of the fluid responsible for the recent acceleration is around $-0.94 \pm 0.1$ [69] and from Eq. (30) we can infer that in a universe dominated by bulk viscous stiff fluid, the corresponding value of the bulk viscous coefficient is $\bar{\zeta}>4$ causing the recent acceleration. Thus the analysis of the evolution of $\omega_{\mathrm{s}}$ also shows that the bulk viscous stiff fluid can replace the conventional dark energy in causing the recent acceleration, for $4<\bar{\zeta}<6$.

\subsubsection{Case $3: \bar{\zeta}>6$}

Equations (8) and (17) can be used in this case too for assessing the behaviors of the Hubble parameter and scale factor. For $\bar{\zeta}>6$ these equations show that the resulting universe will always be accelerating. That is, there is no decelerating epoch at all. When $t \rightarrow \infty$ the universe tends to the de Sitter phase. But when $t-t_{0} \rightarrow-\infty$ the scale factor tends to a finite minimum value (see Fig. 2) instead of zero and it is given as

$$
\lim _{t-t_{0} \rightarrow-\infty} a(t) \equiv a_{\min }=\left(1-\frac{6}{\bar{\zeta}}\right)^{1 / 3} .
$$

The corresponding derivatives $\dot{a}$ and $\ddot{a}$ are zero and hence in this limit the universe becomes an Einstein static universe. As the universe evolves, the scale factor increases monotonically. So there is no big bang in this case and the age of the universe is not properly defined.

The curvature scalar can be obtained using Eq. (15). At $a=a_{\min }$, both $\ddot{a}$ and $H$ are zero, hence the curvature scalar is also zero and it increases as the universe expands, attaining the maximum value $R=\frac{5}{9}\left(H_{0} \bar{\zeta}\right)^{2}$ when $a \rightarrow \infty$. The density of the bulk viscous stiff fluid follows the same behavior as the curvature scalar (see Fig. 1); the density is zero when $a=a_{\min }$ and attains the maximum value $\left(H_{0} \bar{\zeta}\right)^{2} / 12$ as $a \rightarrow \infty$.

\section{Statefinder analysis for $4<\bar{\zeta}<6$}

In the analysis in Sect. 2.1.2 we have concluded that there is a transition from decelerated to accelerated expansion in the recent past when $4<\bar{\zeta}<6$. This gives us hope for the discovery of the recent acceleration of the universe in the context of a universe dominated by a bulk viscous stiff fluid. The behaviors of the scale factor, the $q$ parameter, and the equation of state all show that the bulk viscous stiff fluid is playing the role of dark energy. So we analyze the model using statefinder parameters to compare it with the standard dark energy models. Statefinder parameters [71] are sensitive tools to discriminate various dark energy models, and they are defined as

$r=\frac{\ddot{H}}{H^{3}}-3 q-2$

and

$s=\frac{r-1}{3(q-1 / 2)}$.

Using the equations for the Hubble parameter (8) and the deceleration parameter (26), the $r-s$ parameter equations can be expressed as

$r=\frac{9(6-\bar{\zeta})^{2} a^{-6}}{\left(\bar{\zeta}+(6-\bar{\zeta}) a^{-3}\right)^{2}}+1$

and

$s=\frac{2(6-\bar{\zeta})^{2} a^{-6}}{(6-\bar{\zeta})^{2} a^{-6}-\bar{\zeta}^{2}}$.

The equations show that in the limit $a \rightarrow \infty$ the statefinder parameters $(r, s) \rightarrow(1,0)$, a value similar to the $\Lambda \mathrm{CDM}$ model of the universe, which shows that the model resembles the $\Lambda \mathrm{CDM}$ model in the far future of the evolution of the universe. A plot of the present model in the $r-s$ plane is shown in Fig. 5, for the bulk viscous coefficient $\bar{\eta}=5$, and we also found that the plot for other values of $\bar{\zeta}$ show the same behavior. The plot reveals that the $(r, s)$ trajectory is lying in the region corresponding to $r>1 s<0$, a feature similar to that of the generalized Chaplygin gas model of dark energy [72]. On the other hand in comparison with the holographic dark energy model with event horizon as the IR-cut-off $[73,74]$ whose $r-s$ evolution starts in the region $s \sim 2 / 3, r \sim 1$, and ends on the $\Lambda$ CDM point, the present model starts in the region $r>1, s<0$, and ends on the $\Lambda \mathrm{CDM}$ point in the $r-s$ plane. Equations (34) and (35) show that for $\bar{\zeta}=0,(r, s)=(10,2)$ and for higher values of $\bar{\zeta}$ the $(r, s)$ parameter values decrease. The values of the statefinder parameters for the present stage of the universe dominated by a bulk viscous stiff fluid, corresponding to $a=1(z=0)$, is 


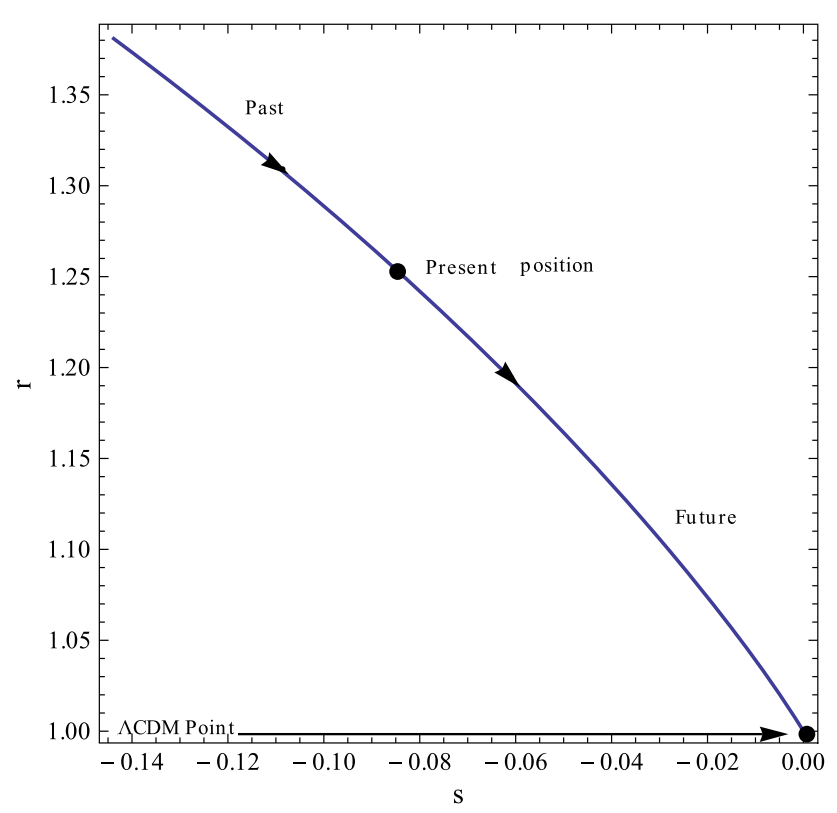

Fig. 5 The $r-s$ plane evolution of the model. The present position of the universe in the plane corresponds to values $\left(r_{0}, s_{0}\right)=(1.25,-0.08)$ is noted. The future evolution of the universe is along the direction as shown by the arrow in the line

$r(a=1)=2\left(1-\frac{\bar{\zeta}}{6}\right)^{2} s(a=1)=\frac{(1-\bar{\zeta} / 6)}{3(1-\bar{\zeta} / 3)}$.

This shows that as $\bar{\zeta}$ increases the present values of $(r, s)$ decrease. In Fig. 5 the present position of the universe is marked and it corresponds to $(r, s)=(1.25,-0.08)$, which is different from the $\Lambda \mathrm{CDM}$ model. This means the model presented here is distinguishably different from the $\Lambda \mathrm{CDM}$ model of the universe.

\section{Entropy and generalized second law of thermodynamics}

Bulk viscosity may be the only dissipative effect occurring in a homogeneous and isotropic universe. Any covariant description of dissipative fluids, where no matter creation takes place, must satisfy the conservation equation,

$T_{; \mu}^{\mu \nu}=0$

where $T^{\mu v}$ is the energy momentum tensor of the fluid in the universe and the semicolon denotes the covariant derivative. The energy momentum tensor in covariant form is given as [75,76],

$T_{\mu \nu}=\rho u_{\mu} u_{\nu}+\left(g_{\mu \nu}+u_{\mu} u_{\nu}\right) p^{\prime}$,

where $u_{\mu}$ is the velocity of the observer who measures the pressure $p^{\prime}$, the form of which is given in Eq. (2). The conservation equation with the above form of the energy momentum tensor will lead to Eq. (6). The bulk viscosity causes the generation of local entropy in the FLRW universe $[75,76]$. The viscous entropy generation in the early universe was studied in Ref. [77]. It has been established that during the evolution of the universe the sum of the entropies of the fluid within the universe and that of the horizon must always be greater than or equal to zero. This important result is known as the generalized second law (GSL) of thermodynamics. The status of the GSL for the flat FLRW universe with matter and a cosmological vacuum was discussed in Ref. [78]. The status of the GSL in a flat universe with viscous dark energy was discussed in Ref. [79], where the authors have shown that the GSL is valid in the FLRW universe with the apparent horizon as the boundary.

In this section we analyze the validity of GSL in the present model of the universe dominated by a bulk viscous stiff fluid with the apparent horizon taken as the boundary of the universe. The GSL can be formally stated as

$\dot{S}_{\mathrm{s}}+\dot{S}_{\mathrm{h}} \geq 0$,

where $S_{\mathrm{s}}$ is the entropy of the stiff fluid and $S_{\mathrm{h}}$ is that of the apparent horizon of the universe. The entropy of the stiff fluid within the horizon of the universe is related to its energy density and pressure through the Gibb's relation [80],

$T \mathrm{~d} S_{\mathrm{s}}=\mathrm{d}\left(\rho_{\mathrm{s}} V\right)+p^{\prime} \mathrm{d} V$,

where $V=4 \pi / 3 H^{3}$ is the volume of the universe within the apparent horizon with radius $r=H^{-1}$ and $T$ is the temperature of the fluid within the horizon. We take the temperature $T=H / 2 \pi$ equal to the Hawking temperature of the horizon with the assumption that the fluid within the horizon is in equilibrium with the horizon, so there is no effective flow of the fluid toward the horizon. Using the dynamical equation and the net pressure in Eq. (2), the time evolution of the entropy of the bulk viscous stiff fluid within the horizon becomes

$\dot{S}_{\mathrm{s}}=\frac{16 \pi^{2} \dot{H}}{H^{3}}-\frac{24 \pi^{2} \dot{H}}{H^{4}}(2 H-\bar{\zeta})$.

The entropy of the apparent horizon is given by the Bakenstein-Hawking formula [81-83],

$S_{\mathrm{h}}=2 \pi A$

where $A=4 \pi H^{2}$ is the area of the apparent horizon. Hence the time rate of the horizon entropy is

$\dot{S}_{\mathrm{h}}=-\frac{16 \pi^{2} \dot{H}}{H^{3}}$.

From Eqs. (41) and (43) the GSL condition given by Eq. (39) is satisfied if

$\dot{H}(\bar{\zeta}-2 H) \geq 0$. 


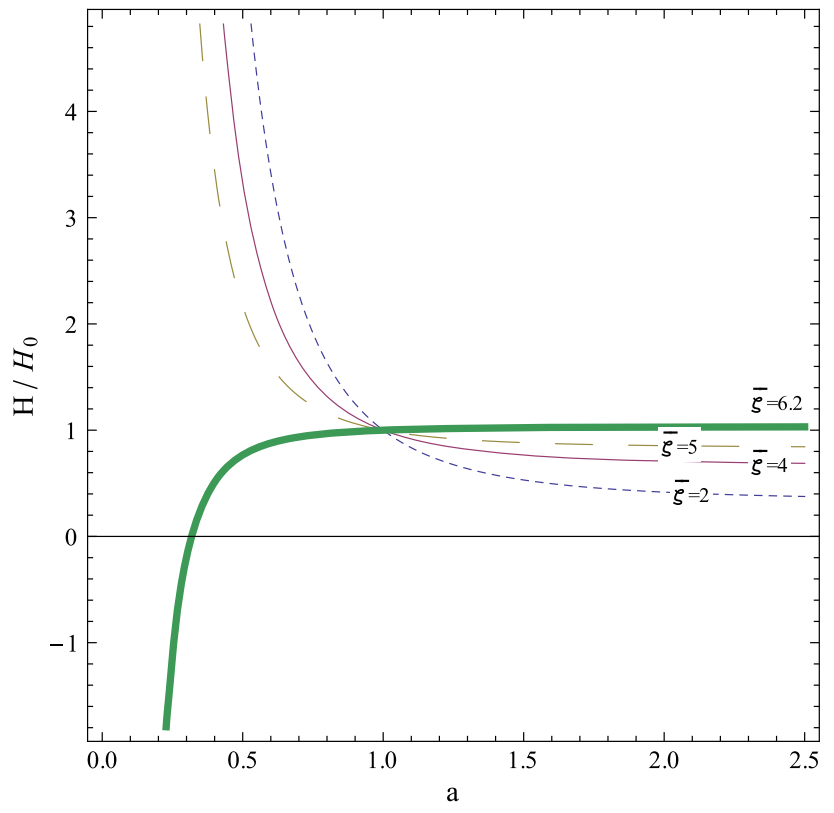

Fig. 6 Evolution of the Hubble parameter with scale factor

Using Eq. (7) the above condition becomes

$H(\bar{\zeta}-2 H)^{2} \geq 0$.

As far as $H$ is positive in an expanding the universe, it is evident that the GSL is satisfied in a bulk viscous stiff fluid dominated universe with the apparent horizon as boundary. From Eq. (8), the required condition for the validity of GSL is

$\left(\bar{\zeta}+(6-\bar{\zeta}) a^{3}\right) \geq 0$.

For $0<\bar{\zeta} \leq 6$ the above condition is fulfilled and consequently the GSL is well satisfied. But when $\bar{\zeta}>6$, the above condition is satisfied only when $a \geq a_{\min }$ as given by Eq. (31) and it is clear from the plot given in Fig. 6 of the Hubble parameter with the scale factor.

\section{Conclusions}

In this paper we present a study of the bulk viscous stiff fluid dominated universe model with a constant bulk viscous coefficient $\bar{\zeta}$. A stiff fluid is an exotic fluid with the equation of state parameter $\omega_{\mathrm{s}}=1$, first studied by Zeldovich [17]. We have analyzed the different possible phases of the model according to the value of the dimensionless bulk viscous parameter $\bar{\zeta}$ taking $\bar{\zeta} \geq 0$. For $\bar{\zeta} \geq 0$ the model predicts an expanding universe in general. For $\bar{\zeta}=0$ the model reduces to a non-viscous stiff fluid dominated universe beginning with a big bang, which is always decelerating with the density varying as $\rho \sim a^{-6}$.
For $0<\bar{\zeta}<6$ the model corresponds to a universe starting with a big bang and undergoing an initial decelerated expansion followed by a transition to an accelerated phase of expansion at later time. For $\bar{\zeta}=4$ the transition from decelerated to accelerated expansion epoch takes place at the present time. For $0<\bar{\zeta}<4$ the transition to the accelerated expansion phase will take place in the future, but for $4<\bar{\zeta}<6$ this transition would have occurred in the past. This shows that the bulk viscous stiff fluid can cause the recent acceleration of the universe. From the behavior of the scale factor we have obtained the age of the universe as $t_{0}-t_{\mathrm{B}}=-\left(2 / H_{0} \bar{\zeta}\right) \ln (1-\bar{\zeta} / 6)$.

We have also studied the evolution of the deceleration parameter $q$ and the equation of state parameter $\omega_{\mathrm{s}}$ for $0<\bar{\zeta}<6$. For $\bar{\zeta}=4$ the deceleration parameter enters the negative region at the present time resulting in a currently accelerated universe. For $\bar{\zeta}<4$ the value of $q$ will enter the negative region in the future. For $4<\bar{\zeta}<6$ it would have entered the negative region in past, implying that the universe made a transition from the decelerated to its accelerated phase in the past. In general $q \rightarrow-1$ as $a \rightarrow \infty$, corresponding to the de Sitter model of the universe. However, for $\bar{\zeta}>6$, $q$ is always negative, implying eternal acceleration without any transition from decelerated to accelerated epoch.

The behavior of $\omega_{\mathrm{S}}$ shows that its value changes from positive to negative when $0<\bar{\zeta}<6$, which implies a transition from decelerated to accelerated epoch. It is always negative when $\bar{\zeta}>6$, which results in an eternally accelerated universe. But irrespective of the value of the viscous coefficient, $\omega_{\mathrm{s}} \rightarrow-1$ as $z \rightarrow-1(a \rightarrow \infty)$. The equation giving the present value of $\omega_{\mathrm{s}}$ indicates that it would be negative today if $\bar{\zeta}>3$, which, however, does not correspond to an accelerating universe. For an accelerating universe $\omega_{\mathrm{s}}<-\frac{1}{3}$, for which $\bar{\zeta}>4$ according to the equation of the present value of $\omega_{\mathrm{s}}$.

Statefinder analysis of the model for the range $4<\bar{\zeta}<6$ predicts the recent acceleration of the universe. The current position of the model in the $r-s$ plane is found to be $\left(r_{0}, s_{0}\right)=$ $(1.25,-0.08)$ which is different from the $\Lambda \mathrm{CDM}$ model. However, as $a \rightarrow \infty$ the statefinder parameters $(r, s) \rightarrow$ $(1,0)$, which corresponds to the $\Lambda \mathrm{CDM}$ point.

When $\bar{\zeta}>6$ we have found that as $\left(t_{0}-t\right) \rightarrow-\infty$ the scale factor tends to a minimum as given by Eq. (31). In this case the model does not have a big bang. The density and the curvature scalar increase as the universe expands and attain their maxima as $a \rightarrow \infty$.

We have analyzed the status the GSL in the present model and found that the GSL of thermodynamics is generally valid with the apparent horizon as the boundary when $0<\bar{\zeta}<6$. But when $\bar{\zeta}>6$ the GSL is satisfied only if the scale factor $a>a_{\min }$ where $a_{\min }$ is given by Eq. (31).

Finally, the question whether the models presented here can successfully replace the conventional dark energy 
models and thus could lead to a unification of the dark sectors can be answered only after a full length analysis of the growth of a linear perturbation and structure formation within these models has been carried out. It is clear that the bulk viscous stiff fluid will behave differently from the cold dark matter regarding linear perturbation growth. The bulk viscous pressure can effectively lead to decay of the density perturbation initially and in the later stages it will effectively damp out the density perturbations [47]. Thus, it may be doubtful whether this model can effectively explain structure formation. However, an exact opinion regarding this can only be obtained by checking whether this model can effectively predict the CMBR temperature fluctuations.

Acknowledgments We thank the referee for the valuable comments. We also thank Prof. M. Sabir, CUSAT, for the discussion and a careful reading of the manuscript.

Open Access This article is distributed under the terms of the Creative Commons Attribution License which permits any use, distribution, and reproduction in any medium, provided the original author(s) and the source are credited.

Funded by SCOAP $^{3}$ / License Version CC BY 4.0.

\section{References}

1. S. Perlmutter et al., Astrophys. J. 517, 565 (1999)

2. A.G. Riess et al., Astrophys. J. 607, 665 (2004)

3. M. Hicken et al., Astrophys. J. 700, 1097 (2009)

4. E. Komatsu et al., Astrophys J. Suppl. 192, 18 (2011). arXiv: 1001.4538

5. D. Larson et al., Astrophys J. Suppl. 192, 16 (2011). arXiv: 1001.4635

6. W.J. Percival et al., Mon. Not. R. Astron. Soc. 401, 2148 (2010). arXiv:0907.1660 [astro-ph.CO]

7. L. Samushia, G. Chen, B. Ratra, arXiv:0706.1963 [astro-ph]

8. Y. Wang, Phys. Rev. D 78, 123532 (2008)

9. L. Samushia, B. Ratra, Astrophys. J. 714, 1347 (2010). arXiv:0905.3836

10. A. Refregier, Ann. Rev. Astron. Astrophys. 41, 645 (2003). arXiv:astro-ph/0307212

11. J.A. Tyson, G.P. Kochanski, I.P. DellAntonio, Astrophys. J. 498 , L107 (1998). arXiv:astro-ph/9801193

12. S. W. Allen, A. C. Fabian, R. W. Schmidt, H. Ebeling, Mon. Not. R. Astron. Soc. 342, 287 (2003). arXiv:astro-ph/0208394

13. F. Zwicky, Helv. Phys. Acta 6, 110 (1933)

14. V.C. Rubin, W.K.J. Ford, Astrophys. J. 159, 379 (1970)

15. V.C. Rubin, N. Thonnard, W.K. Ford, Astrophys. J. 238, 471 (1980)

16. S. Dutta, S.D.H. Hsu, D. Reeb, R.J. Scherrer, Phys. Rev. D 79, 103504 (2009). arXiv:0902.4699 [astro-ph.CO]

17. Ya. B. Zeldovich, Sov. Phys. JETP 14, 11437 (1962)

18. R. Stiele, T. Boeckel, J. Schaffner-Bielich, Phys. Rev. D 81, 123513 (2010). arXiv:1003.2304

19. P. Horava, Phys. Rev. D 79, 084008 (2009). arXiv:0901.3775 [hep-th]

20. G. Calcagni, JHEP 0909, 112 (2009). arXiv:0904.0829 [hep-th]

21. E. Kiritsis, G. Kofinas, Nucl. Phys. B 821, 467 (2009). arXiv:0904.1334 [hep-th]

22. T.P. Sotiriou, M. Visser, S. Weinfurtner, JHEP 0910, 033 (2009). arXiv:0905.2798 [hep-th]
23. C. Bogdanos, E.N. Saridakis, Class. Quant. Grav. 27, 075005 (2010). arXiv:0907.1636 [hep- th]

24. S. Carloni, E. Elizalde, P.J. Silva, Class. Quant. Grav. 27, 045004 (2010). arXiv:0909.2219 [hep-th]

25. G. Leon, E.N. Saridakis, JCAP 0911, 006 (2009). arXiv:0909.3571 [hep-th]

26. A. Ali, S. Dutta, E.N. Saridakis, A.A. Sen, Gen. Relat. Grav. 44, 657 (2012). arXiv:1004.2474 [astro-ph.CO]

27. S. Dutta, E.N. Saridakis, JCAP 1005, 013 (2010). arXiv:1002.3373 [hep-th]

28. L. Fernandez-Jambrina, L.M. Gonzalez-Romero, Phys. Rev. D 66, 024027 (2002). arXiv:gr-qc/0402119

29. N. Dadhich, arXiv:gr-qc/9406034

30. M. Mars, Phys. Rev. D 51, 3989 (1995). arXiv:gr-qc/0201078

31. L. Fernandez-Jambrina, Class. Quant. Grav. 14, 3407 (1997). arXiv:gr-qc/0404017

32. J.D. Barrow, Phys. Lett. B 180, 335 (1986)

33. R. A. Daishev, W. Zimdahl, Class. Quantum Grav. 20, 5017 (2003)

34. S. Dutta, R.J. Scherrrer, Phys. Rev. D 82, 083501 (2010). arXiv:1006.4166 [astro-ph.CO]

35. W. Zimdahl, D.J. Schwarz, A.B. Balakin, D. Pavon, Phys. Rev. D 64, 063501 (2001). arXiv:astro-ph/0009353

36. J. R. Wilson, G. J. Mathews, G. M. Fuller, Phys. Rev. D 75, 043521 (2007) arXiv:astro-ph/0609687 [SPIRES]

37. G.J. Mathews, N.Q. Lan, C. Kolda, Phys. Rev. D 78, 043525 (2008). arXiv:0801.0853 [SPIRES]

38. R. Maartens, Causal thermodynamics in relativity, in Proceedings of Hanno Rund Workshop on Relativity and Thermodynamics, South Africa, 1996 (unpublished) (1996). arXiv:astro-ph/9609119

39. H. Okumura, F. Yonezawa, Phys. A 321, 207 (2003)

40. M. Heller, Z. Klimek, L. Suszycki, Astrophys. Space Sci. 20, 205 (1973)

41. G.L. Murphy, Phys. Rev. D 8, 4231 (1973)

42. M. Heller, Z. Klimek, Astrophys. Space Sci. 33, L37 (1975)

43. I. Waga, R. C. Falcao, R. Chanda, Phys. Rev. D 33, 1839 (1986) [SPIRES]

44. A. Beesham, Phys. Rev. D 48, 3539 (1993)

45. W. Zimdahl, Phys. Rev. D 535483 (1996). arXiv:astro-ph/9601189

46. T. Padmanabhan, S.M. Chitre, Phys. Lett. A 120, 443 (1987)

47. B. Li, J.D. Barrow, Phys. Rev. D 79, 103521 (2009)

48. W.S. Hiplito-Ricaldi, H.E.S. Velten, W. Zimdahl, Phys. Rev. D 82, 063507 (2010)

49. A. Avelino, U. Nucamendi, JCAP 04, 006 (2009)

50. A. Avelino, U. Nucamendi, JCAP 08, 009 (2010)

51. P.S. Wesson, J. Math. Phys. 19, 2283 (1978)

52. E. Masso, R. Rota, Phys. Rev. D 68, 123504 (2003)

53. C. Eckart, Phys. Rev. 58, 919 (1940)

54. L.D. Landau, E.M. Lifshitz, Fluid Mechanics (Addison-Wesley, Reading, 1958)

55. W.A. Hiscock, L. Lindblom, Phys. Rev. D 31, 725 (1985)

56. W. Israel, Ann. Phys. (N.Y.) 100, 310 (1976)

57. W. Israel, J. M. Stewart, Ann. Phys. (N.Y.) 118, 341 (1979)

58. W. Israel, J.M. Stewart, Proc. R. Soc. Lond. A 365, 43 (1979)

59. G. M. Kremer, F. P. Devecchi, Phys. Rev. D 67, 047301 (2003). arXiv:gr-qc/0212046

60. M. Cataldo, N. Cruz, S. Lepe, Phys. Lett. B 619, 5 (2005). arXiv:hep-th/0506153

61. J. C. Fabris, S. V. B. Goncalves, R. de S Ribeiro, Gen. Relat. Grav. 38, 495 (2006). arXiv:astro-ph/0503362

62. M.-G. Hu, X.-H. Meng, Phys. Lett. B 635, 186 (2006). arXiv:astro-ph/0511615

63. J. Ren, X.-H. Meng, Phys. Lett. B 633, 1 (2006)

64. W.A. Hiscock, J. Salmonson, Phys. Rev. D 43, 3249 (1991)

65. D. Pavon, D. Jou, J. Casas-Vazquez, Ann. Inst. Henri Poincare 36, 79 (1982) 
66. WMAP collaboration, G. Hinshaw et al., Five-year Wilkinson microwave anisotropy probe (WMAP) observations: data processing, sky maps \& basic results. Astrophys. J. Suppl. 180, 225 (2009). arXiv:0803.0732

67. E. W. Kolb, M. S. Turner, The Early Universe. Addison-Wesley, California (1990)

68. K. Bamba, S. Capozziello, S. Nijori, S.D. Odintsov, Astrophys. Space Sci. 342, 155 (2012)

69. M. Tegmark, D.J. Eisenstein, M.A. Strauss, D.H. Weinberg, M.R. Blanton et al., Phys. Rev. D 74, 123507 (2006)

70. P. Praseetha, T.K. Mathew, Int. Nat. J. Mod. Phys. D 23, 1450024 (2014)

71. V. Sahni, T.D. Saini, A.A. Starobinsky, U. Alam, JETP Lett. 77, 201 (2003)

72. Y.B. Wu, S. Li, M.H. Fu, J. He, Gen. Relat. Grav. 39, 653 (2007)
73. Z.G. Huang, X.M. Song, H.Q. Lu, W. Fang, Astrophys. Space Sci. 315, 175 (2008)

74. B. Wang, Y. Gong, E. Abdalla, Phys. Rev. D 74, 083520 (2006)

75. S. Weinberg, Gravitation and Cosmology: Principles and Applications of the General Theory of Relativity. Wiley, New York (1971)

76. S. Weinberg, Astrophys. J. 168, 175 (1971)

77. I. Brevik, L.T. Heen, Astrophys. Space Sci. 219, 99 (1994)

78. T.K. Mathew, R. Aiswarya, K.S. Vidya, Eur. Phys. J. C 73, 2619 (2013)

79. K. Karami, A. Sheykhi, N. Sahraei, S. Ghaffari, EPL 93, 29002 (2011)

80. G. Izquierdo, D. Pavon, Phys. Lett. B. 633, 420 (2006)

81. J.D. Bekenstein, Phys. Rev. D 7, 2333 (1973)

82. S.W. Hawking, Commun. Math. Phys. 43, 199 (1975)

83. P.C.W. Davies, Class. Quantum Grav. 5, 1349 (1988) 